\title{
472053 - PROPOFOL DECREASES THE AMPLITUDE OF MOTOR-EVOKED POTENTIALS IN A CONCETRATION-DEPENDENT MANNER
}

\author{
Masashi Uchida, Medicine ${ }^{1}$, Makoto Nishio, Medicine ${ }^{2}$, Syuntaro Hanamura, \\ Medicine $^{2}$, Naoyuki Sizu, Medicine ${ }^{2}$, Noriko Ikeda, Laboratory Medicine ${ }^{3}$, Akihiro \\ Yamauchi, Laboratory Medicine ${ }^{3}$, Sadaaki Nakai, Medicine ${ }^{2}$, Kiyoshi Takeda, \\ PhD,Dr ${ }^{1}$
}

1. Anesthesiology, Fujita Health University, Toyoake, Japan

2. Orthopaedic Surgery, Fujita Health University, Toyoake, Japan

3. Laboratory Medicine, Fujita Health University, Toyoake, Japan

Introduction: Monitoring of descending corticospinal pathways by using motor-evoked potentials (MEPs) has been proven to be useful in preventing neurological deficits during spinal procedures.We reported that propofol markedly decreased the amplitude of MEPs in some patients who had symptoms in upper or lower extremities(1).The mechanisms of it has not proven yet,however this propofol's suppressive effect to MEPs amplitude might happen to every patient.This study aimed to find propofol effect-site concentration (PESC) which gave $50 \%$ decrease in MEPs amplitude in patients whose MEPs monitoring were successful at PESC $2.0 \mu \mathrm{g} / \mathrm{ml}$ and PESC which gave the complete suppression of MEPs facilitation.

Methods: The protocol of this study was approved by the local ethical committee and was performed in a prospective fashion.Written,informed consent was obtained from each patient.Five patients,ASA physical status I-П,undergoing cervical vertebrae surgeries, were included in this study.MEPs were recorded from deltoids and anal sphincters in all patients and MEPs from other two muscles of lower extremities were recorded in accordance with symptoms.After intubation without using muscle relaxant,anesthesia was maintained with air/oxygen and continuous propofol,fentanyl infusion.PESC was simulated by a software developed by Marsh.After the complete elimination of sevoflurane from expiratory breath,MEPs recording was started.When the operation ended,propofol infusion was stopped and MEPs were recorded as PESC decreased.MEPs facilitation was done by transcranial electric stimulation.BIS was monitored in every patient and maintained at 40-60.The regression line $(\mathrm{PESC}(\mu \mathrm{g} / \mathrm{ml}) \mathrm{vs}$. MEP amplitude $(\mu \mathrm{V})$ )was drawn for each muscle by using SigmaPlot 10.0.1 (HULINKS) and the MEP amplitude at PESC 2.0 $\mu \mathrm{g} / \mathrm{ml}$,PESC which gave 50\% decrease in MEP amplitude,and PESC which gave the complete suppression of MEP facilitation were obtained from the regression line.

Results: MEPs were analyzed in seven muscles in five patients.In every muscle propofol decreased MEPs amplitude in a concentration-dependent manner and PESC which gave $50 \%$ decrease of MEPs amplitude was 3.73 \pm 0.75 (mean \pm SD) $\mu \mathrm{g} / \mathrm{ml}$ and PESC which gave the complete suppression of MEPs facilitation was $6.03 \pm 1.93 \mu \mathrm{g} / \mathrm{ml}$.

Discussion: Scheufler et.al. reported that propofol decreased MEPs amplitude in a concentration-dependent manner and PESCs more than $2 \mu \mathrm{g} / \mathrm{ml}$ markedly decreased MEPs amplitude (2).In their study PESC which gave 50\% decrese in MEPs amplitude was supposed to be $3.0-5.0 \mu \mathrm{g} / \mathrm{ml}$ and PESC which gave the complete suppression of 
MEPs facilitation was supposed to be more than $6.0 \mu \mathrm{g} / \mathrm{ml}$. The values which we obtained from this study were almost consistent with their results.

References: (1)Anesthesiology 2007;107:A1500 (2)Anesthesia and Analgesia 2002;94:907-912 